\title{
Surface tension and interfacial fluctuations in $d$-dimensional Ising model
}

\author{
J. Kaupužs * \\ Institute of Mathematics and Computer Science, University of Latvia \\ 29 Rainja Boulevard, LV-1459 Riga, Latvia
}

October 17, 2018

\begin{abstract}
The surface tension of rough interfaces between coexisting phases in 2D and 3D Ising models are discussed in view of the known results and some original calculations presented in this paper. The results are summarised in a formula, which allows to interpolate the corrections to finite-size scaling between two and three dimensions. The physical meaning of an analytic continuation to noninteger values of the spatial dimensionality $d$ is discussed. Lattices and interfaces with properly defined fractal dimensions should fulfil certain requirements to possibly have properties of an analytic continuation from $d$-dimensional hypercubes. Here 2 appears as the marginal value of $d$ below which the $(d-1)-$ dimensional interface splits in disconnected pieces. Some phenomenological arguments are proposed to describe such interfaces. They show that the character of the interfacial fluctuations at $d<2$ is not the same as provided by a formal analytic continuation from $d$-dimensional hypercubes with $d \geq 2$. It, probably, is true also for the related critical exponents.
\end{abstract}

Keywords: Ising model, surface tension, fractal dimension

\section{Introduction}

The phase coexistence and surface tension of fluctuating interfaces is an object of extensive theoretical studies. It covers exact results for two-dimensional Ising and solid-on-solid (SOS) models [1, low-temperature series analysis of 3D Ising model [2, studies within the cappilary wave approximation at arbitrary spatial dimension $d$ [3], a general phenomenological description [4], as well as Monte Carlo studies of surface tension in 3D Ising model [5, 6]. We recommend the review papers [7, 8] for further references.

In this paper first we will briefly discuss the existing results for rough interfaces in $2 \mathrm{D}$ and $3 \mathrm{D}$ Ising models, completing them by some original calculations. Then we will discuss the spatial dimensionality $d$ as a continuous parameter from a purely formal point of view, as well as linking noninteger $d$ values to lattices with certain fractal dimension. The latter consideration suggests that 2 is a special marginal value of $d$.

${ }^{*}$ E-mail: kaupuzs@latnet.lv 


\section{Surface tension of 2D Ising model}

Consider the Ising model with the Hamiltonian $H$,

$$
\frac{H}{k_{B} T}=-\beta \sum_{\langle i j\rangle} s_{i} s_{j}
$$

where $k_{B}$ is the Boltzmann constant, $T$ is the temperature, $s_{i}= \pm 1$ are the spin variables, and $\beta$ is the coupling constant describing the ferromagnetic interaction between all pairs $\langle i j\rangle$ of the neighbouring spins. In the low-temperature phase at

$\beta>\beta_{c}$, where $\beta_{c}=\frac{1}{2} \ln (1+\sqrt{2})$ is the critical coupling, certain interfacial structure can be imposed by appropriate boundary conditions.

The surface tension of inclined interfaces in 2D Ising model has been considered in [1, 4. As defined in 1], the spins are located at lattice points $x=0,1,2, \ldots, L$ and $y= \pm \frac{1}{2}, \pm \frac{3}{2}, \ldots, \pm\left(M-\frac{1}{2}\right)$. The interface which makes a mean angle $\theta$ with $x$ axis is forced by the boundary conditions (see Fig. 1 in [1, 4] $s(x, \pm(M-1 / 2))= \pm 1$, $s(0, y>0)=1, s(0, y<0)=-1, s(L, y>m)=1, s(L, y<m)=-1$. Hence, the endpoints of the interface are pinned at $x=0 ; y=0$ and $x=L ; y=m$, and $\tan \theta=m / L$. The quantity of interest is the partition function $Z(m, L ; M)$ of the lattice with such an interface, normalized to the partition function of the lattice without the interface, for which all the boundary spins are fixed positive.

The surface tension $\sigma(\theta, L ; M)$ is defined as [1]

$$
\sigma(\theta, L ; M)=-\frac{\cos \theta}{L} \ln Z(L \tan \theta, L ; M) .
$$

The bulk surface tension at $\theta=0$, i. e., $\tau \equiv \sigma(0, \infty, \infty)>0$ and the surface stiffness $\kappa>0$ are defined according to

$$
\frac{\sigma(\theta, \infty, \infty)}{\cos \theta}=\tau+\frac{1}{2} \kappa \theta^{2}+O\left(\theta^{4}\right)
$$

The influence of the size $M$ decreases exponentially at $L \rightarrow \infty$ when $M=$ $O(L)$ 1]. Based on exact formula for $Z(m, L) \equiv Z(m, L ; \infty)$, it has been found in [1] that

$$
Z(m, L) \simeq \exp \left(-\tau_{L} L\right)\left(\frac{\kappa_{L}}{2 \pi L}\right)^{1 / 2} \exp \left(\frac{-\kappa_{L} m^{2}}{2 L}\right)
$$

holds for large $L$ with

$$
\tau_{L}=\tau+\frac{a}{L}+o\left(\frac{1}{L}\right), \quad \kappa_{L}=\kappa+\frac{b}{L}+o\left(\frac{1}{L}\right) .
$$

Here $\tau=2\left(\beta-\beta^{*}\right)$, where $\beta^{*}$ is the dual coupling defined by $\exp (-2 \beta)=\tanh \beta^{*}$, $\kappa=\sinh \tau, a=2 \beta$, and $b=\frac{1}{2}\left[\sinh ^{2} 2 \beta^{*}+\sinh ^{2} \tau+3 \cosh \tau\right]$. The square root term in (4) is the normalization factor of the Gaussian distribution.

Hence the surface tension $\tilde{\sigma}(0, L) \equiv \sigma(0, L ; \infty)$ is [1]

$$
\tilde{\sigma}(0, L)=\tilde{\sigma}(0, \infty)+\frac{\ln L}{2 L}+\frac{a-\ln \left[(\kappa / 2 \pi)^{1 / 2}\right]}{L}+o\left(\frac{1}{L}\right) .
$$


Note that the universal logarithmic correction term $\frac{1}{2} \ln L / L$ comes from the normalization factor in (4). Similar corrections to scaling appear also in the case, discussed below, where the interface is forced by anti-periodic boundary conditions along one of the axes, the boundary conditions being periodic along the other axis.

An exact expression for the partition function of a finite-size $2 \mathrm{D}$ lattice on a torus with arbitrary coupling constants between each pair of neighbouring spins has been reported in 9] obtained by the loop counting method and represented by determinants of certain transfer matrices. In the standard 2D Ising model with only one common coupling constant $\beta$ these matrices can be diagonalized easily, using the standard techniques [10]. Besides, the loop counting method can be trivially extended to the cases with antiperiodic or mixed boundary conditions. It is necessary only to mention that each loop gets an additional factor -1 when it winds round the torus with antiperiodic boundary conditions. We consider the partition functions $Z_{p p}, Z_{a a}, Z_{a p}, Z_{p a}$. In this notation the first index refers to $x$ axis, and the second one - to $y$ axis; $p$ means periodic and $a$ - antiperiodic boundary conditions. Thus, for the lattice with $x=1,2, \ldots, N$ and $y=1,2, \ldots, L$, we obtain the following exact expressions:

$$
\begin{aligned}
Z_{p p} & =\left(Q_{1}+Q_{2}+Q_{3}-Q_{0}\right) / 2 \\
Z_{a p} & =\left(Q_{0}+Q_{1}+Q_{3}-Q_{2}\right) / 2 \\
Z_{p a} & =\left(Q_{0}+Q_{1}+Q_{2}-Q_{3}\right) / 2 \\
Z_{a a} & =\left(Q_{0}+Q_{2}+Q_{3}-Q_{1}\right) / 2
\end{aligned}
$$

where $Q_{0}$ is the partition function represented by the sum of the closed loops on the lattice, as consistent with the loop counting method in [10], whereas $Q_{1}, Q_{2}$, and $Q_{3}$ are modified sums with additional factors $\exp (\Delta x \cdot i \pi / N+\Delta y \cdot i \pi / L), \exp (\Delta x \cdot i \pi / N)$, and $\exp (\Delta y \cdot i \pi / L)$, respectively, related to each change of coordinate $x$ by $\Delta x= \pm 1$, or coordinate $y$ by $\Delta y= \pm 1$ when making a loop. The standard manipulations [10] yield

$$
\begin{aligned}
& Q_{i}=2^{N L} \prod_{q_{x}, q_{y}}\left[\cosh ^{2}(2 \beta)-\sinh (2 \beta)\right. \\
& \left.\times\left(\cos \left[q_{x}+\left(\delta_{i, 1}+\delta_{i, 2}\right) \frac{\pi}{N}\right]+\cos \left[q_{y}+\left(\delta_{i, 1}+\delta_{i, 3}\right) \frac{\pi}{L}\right]\right)\right]^{1 / 2},
\end{aligned}
$$

where the wave vectors $q_{x}=(2 \pi / N) \cdot n$ and $q_{y}=(2 \pi / L) \cdot \ell$ run over all the values corresponding to $n=0,1,2, \ldots, N-1$ and $\ell=0,1,2, \ldots, L-1$. Eq. (8) represents an analytic extension from small $\beta$ region $[9$. The correct sign of square roots is defined by this condition, and all $Q_{i}$ are positive except for $Q_{0}$, which vanishes at $\beta=\beta_{c}$ and becomes negative at $\beta>\beta_{c}$. The sign-alternating factor with $q_{x}=q_{y}=0$ can be written as $1-\sinh (2 \beta)$. In the case of the periodic boundary conditions, each loop of $Q_{0}$ has the sign $(-1)^{m+a b+a+b}$ [9], where $m$ is the number of intersections, $a$ is the number of windings around the torus in $x$ direction, and $b-$ in $y$ direction. The correct result for $Z_{p p}$ is obtained if each of the loops has the sign $(-1)^{m}$. Eq. (7) for $Z_{p p}$ is then obtained by finding such a linear combination of quantities $Q_{i}$ which ensures the correct weight for each kind of loops. Eqs. (7) for $Z_{a a}, Z_{a p}$, and $Z_{p a}$ are obtained in an analogous way. 
The surface tension is given by the Onsager's ansätz

$$
\sigma(L, N)=L^{-1} \ln \left(N Z_{p p} / Z_{a p}\right)
$$

where the size $N$ in $x$ direction is included since, due to the translation symmetry, each interface configuration has $N$ equivalent copies obtained by shifting along the $x$ axis. It means that we take only one of the $N$ equivalent copies for the interfacial partition function $N^{-1} Z_{a p} / Z_{p p}$.

We have analysed the corrections to scaling for $\sigma(L, N)$ numerically. Considering a trial function of the form

$$
\sigma(L, L) \simeq \tau+A \ln L / L+B / L
$$

with $\tau=2\left(\beta-\beta^{*}\right)$, the coefficients $A$ and $B$ have been evaluated by fitting the calculated values of $\sigma(L, L)$ and $\sigma(2 L, 2 L)$. We have observed that the obtained effective coefficient $A(L)$ converges almost linearly in $1 / L$ to certain asymptotic value, whereas $B(L)$ plot looks more linear in the scale of $\ln L / L$. It means that

$$
\begin{aligned}
& A(L) \simeq A(\infty)+C_{A} / L \\
& B(L) \simeq B(\infty)+C_{B} \ln L / L
\end{aligned}
$$

hold with some constants $C_{A}$ and $C_{B}$. Our values of $A(L)$, computed at $\beta=$ 0.5, are $A(8) \simeq 0.62206, A(16) \simeq 0.56727, A(24) \simeq 0.54638, A(32) \simeq 0.53546$, and $A(40) \simeq 0.52876$. The corresponding values of $B(L)$ are $1.21819,1.37012$, $1.43998,1.48036$, and 1.50687 . We have extracted from these numbers the following estimates: $A=A(\infty)=0.502 \pm 0.011, C_{A}=1.07 \pm 0.20, B=B(\infty)=1.659 \pm 0.015$, and $C_{B}=-1.65 \pm 0.11$. These values have been obtained by fitting $A(L)$ and $B(L)$ to (11) at $L=32,40$, and the discrepancies between the estimates at $L=32,40$ and $L=8,16$ have been assumed as the error bars, indicating the range of possible deviations from the true asymptotic values. According to the observed monotonous behaviour of the coefficients, estimated from (11) for each pair of sizes $L$ and $L-8$, these deviations, most probably, are positive for $A$ and $B$ and negative for $C_{A}$ and $C_{B}$. Our calculations show that $A$, likely, has the same universal value $1 / 2$ as the coefficient at $\ln L / L$ in ([6), whereas $B$ differs from the corresponding coefficient in (6) , the latter being $B^{\prime} \simeq 2.653678$ at $\beta=0.5$. Besides, the observed deviations from the asymptotic law (10) are characterised by a remainder term $\sim \ln L / L^{2}$, which is compensated by $A \rightarrow A(L)$ and $B \rightarrow B(L)$. Assuming that $A(\infty)=1 / 2$, the estimation of coefficient $C_{A}$ can be improved. From the first equation of (11), then we obtain $C_{A}=1.15 \pm 0.08$ at $L=40$. Here the discrepancy with our previous value has been put for the error bars.

These calculations for system sizes up to $2 L=80$ have been performed by double-precision FORTRAN codes. In this case computations at larger system sizes become problematic due to the rounding errors: it is necessary for calculation of $Z_{a p}$ to extract from the linear combination of $Q_{i}$ a quantity, which is exponentially small relative to $\left|Q_{i}\right|$. Therefore, a more precise estimation of the asymptotic values requires a computation with substantially larger number of digits. 


\section{Surface tension of 3D Ising model}

According to the phenomenological description provided in [4], a relation similar to (4) holds for rough interfaces (above the roughening transition temperature $T_{R}$ and below the bulk critical temperature $T_{c}$ ) also in three dimensions. Note that the interface is always rough, i. e., not pinned by the underlaying lattice structure, in two dimensions at nonzero temperature [4, 7.

Consider a $d$-dimensional $(d=2,3) N \times L^{d-1}$ lattice, where $N$ is the linear size in the direction perpendicular to the interface (when $\theta=0$ ). In analogy to [4,

$$
\begin{aligned}
Z(m, L) & \simeq \exp \left(-\tau_{L} L^{d-1}\right) R(L, d) \exp \left(-L^{d-1} \kappa_{L} \theta^{2}\right) \\
& \simeq \exp \left(-\tau_{L} L^{d-1}\right) R(L, d) \exp \left(-L^{d-3} \kappa_{L} m^{2}\right)
\end{aligned}
$$

is expected for the partition function $Z(m, L)$ of an inclined interface with small tilt angle $\theta \simeq m / L$, large $L$, and $N=O(L)$, where $\tau_{L}$ and $\kappa_{L}$ are the finite-size observables of the bulk surface tension $\tau$ and the stiffness coefficient $\kappa$. According to the arguments provided in [4], $R(L, d)$ should behave like the normalization factor of the Gaussian distribution, i. e.,

$$
R(L, d) \sim\left(L^{d-3} \kappa_{L} / 2 \pi\right)^{1 / 2}
$$

should hold for small values of $L^{d-3} \kappa_{L} / 2 \pi$. Eqs. (12) and (13) coincide with (44) at $d=2$ and with Monte Carlo (MC) simulation results for tilted interfaces in three dimensions [5].

If the interface is forced by antiperiodic boundary conditions along one of the axes (where the size is $N$ ) and periodic boundary conditions along the other axes, then its mean slope is zero, therefore the surface tension

$$
\sigma(L, N)=L^{1-d} \ln \left(N Z_{p p} / Z_{a p}\right)
$$

should be more or less consistent with $\sigma=-L^{1-d} \ln Z(0, L)$ calculated from Eqs. (12) and (13) at $\theta=0$. The partition functions $Z_{p p}$ and $Z_{a p}$ in (14) have the same meaning as before, only the second index now refers to all axes aligned parallel to the interface.

The surface tension (14) in 3D case has been properly studied by Monte Carlo simulations in [6] by means of the thermodynamical integration of the interfacial energy. It has been found that the surface free energy $F_{s}=L^{2} \sigma(L, N)$ is well described by the expression of the Gaussian capillary wave theory [3]

$$
F_{s} \simeq C_{s}+\sigma L^{2}
$$

where $C_{s}=G-\frac{1}{2} \ln \sigma$ with $G \approx 0.29$ holds near the critical point. Eq. (15) is consistent with (12) and (13), where the $\frac{1}{2} \ln \sigma$ term comes from (13), taking into account that $\kappa \propto \sigma$ holds at $\beta \rightarrow \beta_{c}$. Contrary to the 2D case, now the leading correction to scaling for $\sigma$ is $\sim 1 / L^{2}$, as consistent with $\tau_{L}=\tau+O\left(1 / L^{2}\right)$, and the logarithmic correction is absent since $d-3=0$ vanishes in (13).

The "endpoint" correction of order $O(1 / L)$ is expected in the case of inclined interfaces (in 3D lattice) considered in [4] due to the direct influence of the fixed 
boundary spins. In the case of (14) such a correction apparently is absent according to [6. We have verified also via direct simulation of the partition functions $Z_{p p}$ and $Z_{a p}$ by the multicanonical Monte Carlo sampling method [11] that $\sigma(L, L)$ at $\beta=0.3$ well coincides with $\sigma(L, L)=\sigma(\infty, \infty)+C_{s} / L^{2}$ law within $L \in[6 ; 16]$.

\section{A formal generalisation to continuous dimension $d$}

The relation (12) is quite general and has to be true for any natural $d \geq 2$ to provide finite values of bulk surface tension and stiffness, the only question is about the specific form of prefactor $R(L, d)$ [4. Besides, it is possible to consider the spatial dimension $d$ in (12) and (13) as a continuous parameter within $1<d \leq 3$. The $d=3$ case is marginal for the normalization factor (13), since the width of the distribution over $m$ in (12) is diverging in the thermodynamic limit at $d<3$ and becomes finite at $d=3$. Due to the latter fact, Eq. (13) at $d=3$, likely, is valid only in vicinity of the bulk critical point, where the distribution width is large.

An approximation for the case where the interface is forced by the mixed boundary conditions (antiperiodic in one direction, periodic - in other directions) is obtained by setting $\theta=0$. The MC results discussed in Sec. 3 suggest that for this kind of boundary conditions the corrections to scaling of the kind $1 / L$, which appear in two dimensions, have to be dechipered in general as $1 / S$ corrections, where $S=L^{d-1}$ is the interface area. Thus, the surface tension $\sigma(L, N)$ of the $N \times L^{d-1}$ lattice with $N=O(L)$ is expected to be

$$
\sigma(L, N)=\sigma(\infty, \infty)+\frac{3-d}{2} \frac{\ln L}{L^{d-1}}+O\left(L^{1-d}\right)
$$

for $d \leq 3$. It allows to interpolate between two and three dimensions. Our further consideration shows that the continuation below $d=2$ is problematic, if one tries to relate it to real physical systems.

\section{Physical interpretation of continuous dimension $d$}

To give some physical meaning to (12), (13), and (16) at a noninteger $d$, one has to relate these formulae to some really existing lattices. We will consider lattices with suitably defined fractal dimension like in [12. Such lattices with interfaces between the coexisting phases, probably, should meet a lot of requirements to be considered in some sense as analytic continuations from natural $d$.

In our further consideration it is suitable to define the interface of a given spin configuration as a set of interfacial spins located near the phase-separation border. We denote by $\Lambda_{S}$ the subset of lattice sites where these spins are located. For an arbitrary lattice, we consider the graph-theoretic distance dist $(x, y)$ between the sites $x$ and $y$, which is defined as the minimum number of bonds in $\Lambda_{b}$ that one needs to connect $x$ and $y$. Here $\Lambda_{b}$ is the set of bonds between the directly interacting neighbouring spins. We denote by $N_{R}(x)$ the number of lattice sites inside a sphere of radius $R$ centered at $x$, i. e., the number of those sites $y$ for which $\operatorname{dist}(x, y)<R$ holds. In analogy, $n_{R}(x)$ is defined as the number of interfacial sites $y \in \Lambda_{S}$ inside the sphere of radius $R$ centered at $x \in \Lambda_{S}$. 
In the thermodynamic limit the $d$-dimensional hypercubes $(d=2,3,4, \ldots)$ with $(d-1)$-dimensional interfaces have certain essential properties, listed below.

(i) The number of bonds in $\Lambda_{b}$ connected to one lattice site is bounded uniformly (for all lattice sites) from above by some positive constant.

(ii) The lattice has certain dimension $d$, defined as

$$
d=\lim _{R \rightarrow \infty} \frac{\ln N_{R}(x)}{\ln R},
$$

which holds for all $x$.

(iii) The interface has certain dimension $d_{s}$, defined as

$$
d_{s}=\lim _{R \rightarrow \infty} \frac{\ln n_{R}(x)}{\ln R},
$$

which holds for all $x \in \Lambda_{S}$, and this dimension is equal to $d-1$.

(iv) By any physically senseful definition of the set of interfacial sites $\Lambda_{S}$, there exists a subset $\Lambda_{s} \subseteq \Lambda_{S}$ of these sites, which forms an infinitely large connected cluster. In other words, the main body of the interface is connected.

There is no reason to expect that lattices and interfaces with noninteger fractal dimension have properties of an analytic continuation from the hypercubes with integer $d$ if any of these requirements is violated.

The following lemma is relevant for our further considerations.

Lemma - If the interface has certain dimension $d_{s}$ such that (18) holds for all $x \in \Lambda_{S}$, and there exists a subset $\Lambda_{s} \subseteq \Lambda_{S}$ of the interfacial sites which form an infinitely large connected cluster, then $d_{s} \geq 1$.

Proof. Choose $x, y \in \Lambda_{s}$ at a distance $\operatorname{dist}(x, y)=R$. By definition of connected cluster, there exists a path connecting $x$ and $y$ by bonds of $\Lambda_{b}$ such that all sites of this path $y_{i}$ with $i=0,1,2, \ldots$, where $y_{0} \equiv x$, belong to $\Lambda_{s}$. Obviously, $\operatorname{dist}\left(x, y_{i}\right)$ reaches $R$ for the first time at some $i=i_{0}$. By definition of the distance, $\operatorname{dist}\left(x, y_{i}\right) \leq i$ holds, so that $i_{0} \geq R$. Thus, there exists at least $R$ sites $y_{i} \in \Lambda_{s}$ with $i=0,1,2, \ldots, i_{0}-1$ such that $\operatorname{dist}\left(x, y_{i}\right)<R$, i. e., $n_{R}(x) \geq R$ holds. Since the cluster is infinitely large, we can choose unlimitedly large $R$. Hence

$$
d_{s}=\lim _{R \rightarrow \infty} \frac{\ln n_{R}(x)}{\ln R} \geq \lim _{R \rightarrow \infty} \frac{\ln R}{\ln R}=1,
$$

which proves the lemma.

According to this lemma, $d=2$ is the lower marginal value of dimension $d$ at which properties (ii) to (iv) still can be satisfied simultaneously. Hence, at $d<2$ the $(d-1)$-dimensional interface cannot contain infinitely large connected clusters, i. e., it splits in disconnected finite-size pieces. Thus, if we would choose $x \in \Lambda_{S}$ and look for the interfacial structure within a sphere $\operatorname{dist}(x, y)<R$, we would see infinitely many disconnected pieces at $R \rightarrow \infty$. Following the consideration we have used to prove the lemma, it is easy to realise that the minimum number of bonds, 
which are necessary to connect all these pieces together, exceeds infinitely many times the number of interfacial sites inside such a sphere at $R \rightarrow \infty$. It implies that, on large enough scales, the interface is essentially disconnected (further referred as frustrated) for any $d<2$ irrespective to that how small is $\epsilon=2-d>0$.

Lattices with $1<d<2$ are, e. g., Sierpiński carpets (see Fig. 2 in [13]) which, however, do not really have the interfacial properties of an analytic continuation from $d$-dimensional hypercubes. A crossection line in this case consists of disconnected pieces, distributed in a fractal way, as we have discussed already. However, if the interface would be induced by appropriate boundary conditions, then its fractal dimension would be $d_{s}<d-1$ rather than $d-1$, since the minimum of free energy corresponds more or less to the minimal crossection with $d_{s}<d-1$. In this aspect, some random (statistical) lattices, which cannot be split in a special way, could be better candidates to mimic an analytic continuation from integer $d$.

\section{Fluctuations of a frustrated interface at $d<2$}

Since the $(d-1)$-dimensional interface becomes frustrated (disconnected) at $d<2$, a formal analytic continuation from $d$-dimensional hypercubes with $d \geq 2$ hardly can be applied to describe it. The disconnected pieces can relatively freely move with respect to each other within some range allowed by the lattice structure, which is a qualitatively new feature as compared to connected interfaces. One may expect that it gives an extra contribution to the interfacial entropy. The pieces of the frustrated interface typically has to be located in such a way to make the narrowest connections between the coexisting phases, i. e., to minimize the free energy. Therefore, on larger scales, the fluctuations in a random lattice are expected to be jump-like, where the pieces of interface are moved from one set of narrow places to another. This interpretation becomes rather clear in a particular case of randomized Sierpiński carpets, obtained by cutting out of the 2D lattice holes of different random shapes (starting from larger holes, then, hierarchially, smaller and smaller holes). The structure of such a lattice with suitable fractal dimension $1<d<2$ consists of a set of holes with "bridges" in between, when looking on any scale. The places, where the pieces of the interface most probably can be located, correspond to the narrowest crossections of these "bridges". We include a randomization, since it eventually could be helpful to mimic essential properties of $d$-dimensional hypercubes, as discussed at the end of Sec. [5]

We propose some phenomenological arguments to describe the above discussed fluctuations of a frustrated interface in a random lattice. In this consideration the fractal dimension of the interface has to be $d_{s}<1$, but not necessarily $d-1$. On a phenomenological level of description, one can introduce a subset of lattice sites $\Omega$, where the interface most probably can be located. It means that only the relevant spin configurations are considered such that $\Lambda_{S} \subseteq \Omega$, which correspond to local minima of free energy. An essential quantity is the probability $Q(m)$ that a local displacement of the interface by a distance $m$ is "allowed" by the lattice structure, i. e., that it corresponds to $\Lambda_{S} \rightarrow \Lambda_{S}^{\prime}$ where $\Lambda_{S}, \Lambda_{S}^{\prime} \subseteq \Omega$. The displacement measured from $x \in \Lambda_{S}$ can be defined as the minimal distance from $x$ to some $x^{\prime} \in \Lambda_{S}^{\prime}$, i. e., $m(x)=\inf _{x^{\prime} \in \Lambda_{S}^{\prime}} \operatorname{dist}\left(x, x^{\prime}\right)$. The probability $Q(m)$ then is $Q(m)=N_{S}^{-1} \sum_{x \in \Lambda_{S}} I(m, x)$, 
where $N_{S}$ is the number of elements in $\Lambda_{S}$ and $I(m, x)$ is the indicator function. It has the value $I(m, x)=1$ if there exists $\Lambda_{S}^{\prime} \subseteq \Omega$ such that $\inf _{x^{\prime} \in \Lambda_{S}^{\prime}} \operatorname{dist}\left(x, x^{\prime}\right)=m$ holds, and $I(m, x)=0$ otherwise. Since the distribution of the interfacial sites around an arbitrarily choosen $x \in \Lambda_{S}$ is characterised by certain fractal dimension $d_{s}$, the distribution of the places $x^{\prime} \in \Omega$, where the interface can be eventually located, and hence the distribution of those values of $m$ for which $I(m, x)=1$ holds also should be characterised by some fractal dimension. Thus, the number of such values of $m$ within $[0 ; M]$ has to increase like $M^{d_{s}^{\prime}}$ at $M \rightarrow \infty$, where $d_{s}^{\prime}<1$ is a fractal dimension. Hence, the expected asymptotic behaviour of $Q(m)$ for large $m$ is $Q(m) \sim m^{d_{s}^{\prime}-1}$. It is true for a frustrated interface at $d<2$. To the contrary, $Q(m) \equiv 1$ corresponds to regular lattices with $d=2,3,4, \ldots$, where the displacements of the interface are quasy-continuous.

Let us assume that the interface is someway pinned at one its point $x \in \Lambda_{S}$ (we may consider this as a constraint for the spin configurations allowed) and consider the probability distribution function $\mathcal{P}(m, L)$ over the displacements $m$ of the interface from its energetically most preferable position, measured at some point $y \in \Lambda_{S}$ at a distance $L=\operatorname{dist}(x, y)$ from $x$. Considering the limit $L \rightarrow \infty$, it is suitable to make an averaging over the set of lattice sites $y$ obeing the relation $|\operatorname{dist}(x, y)-L|<$ $\varepsilon L$, where $\varepsilon$ is small and positive. In this case the density of the local minima of free energy is given by $Q(m)$. A question arises whether or not the probability distribution over the "allowed" states, which correspond to these local minima, can be characterised by certain stiffness coefficient $\kappa_{L}$. If not, then it already means that the fluctuations of the frustrated interface cannot be described by a formula similar to (12). If yes, then an analogous formula reads

$$
\overline{\mathcal{P}}(m, L) \sim Q(m) \exp \left(-L^{d_{s}-2} \kappa_{L} m^{2}\right) .
$$

It is supposed that first the normalized probability distribution function $\mathcal{P}(m, L)$ is found for each individual $y$ and then the distribution $\overline{\mathcal{P}}(m, L)$ is calculated by an averaging over $y$. Besides, an $m$-independent prefactor is omitted in (19). The validity of (19) is restricted to a region $L^{d_{s}-2} \kappa_{L} m^{2}<C$, where $C$ is some constant, to ensure that $\mathcal{P}(m, L)$ is not essentially influenced by relatively small variations in the distance $\operatorname{dist}(x, y)$. This equation agrees with (12) at $Q(m) \equiv 1$. The latter relation can be valid for a connected interface at $d \geq 2$. However, as we have discussed already, $Q(m) \sim m^{d_{s}^{\prime}-1}$ with $d_{s}^{\prime}<1$ is expected for large $m$ in our case of $d<2$ and $d_{s}<1$, where the interface is necessarily disconnected or frustrated (cf. the Lemma). Hence, these arguments suggest that, in any case, the character of the interfacial fluctuations changes qualitatively at $d<2$, as compared to a formal analytic continuation from $d$-dimensional hypercubes with $d \geq 2$. Thus, a formal extension of (16) to $d<2$, likely, has no physical meaning.

\section{Problem of an analytic continuation of the critical exponents}

As shown in Secs. [5 and [6] $d=2$ is a special lower marginal value of $d$ as regards the behaviour of the interface between the coexisting phases. The bulk critical 
behaviour results from a competition of large coexisting domains having opposite sign of the mean magnetisation. Therefore the bulk critical behaviour should be influenced by the interfacial structure. Besides, the critical exponent of the surface tension $\mu$ is related to the bulk critical exponents via $\mu+\nu=2-\alpha=d \nu$ [14. Hence, one can expect that 2 is a special marginal value of $d$ also for these critical exponents. It would mean that an analytic continuation of both interface and bulk critical exponents from $d \geq 2$ ( or $d=2,3$ ) to $d<2$ is only formal, like a continuation from $d<4$ to $d>4$ (above the upper critical dimension $d=4$ ). In this case no appropriate family of lattices could be found, providing the critical exponents as (almost) continuous functions of $d$ in agreement with such an analytic continuation.

A family of fractal lattices, which allows to treat $d$ as a continuous parameter in exact recurrence relations, has been considered in [15. However, these lattices are not quite appropriate to mimic an analytic continuation from $d$-dimensional hypercubes. A particular problem is that the number of bonds connected to one lattice site is not bounded in the thermodynamic limit (i. e., the property (i) is violated).

\section{Conclusions}

1. The surface tension of rough interfaces in $2 \mathrm{D}$ and $3 \mathrm{D}$ Ising models has been discussed. The known results have been completed by some original calculations. In summary, a formula is given [Eq. (16)], which allows to interpolate the corrections to finite-size scaling between two and three dimensions.

2. It has been proven that 2 is the marginal value of $d$ below which the $(d-1)$-dimensional interface between the coexisting phases becomes essentially disconnected or frustrated.

3. Some phenomenological arguments have been proposed to describe the fluctuations of such frustrated interfaces. They show that 2 is a special value of the dimension $d$ such that the interfacial properties at $d<2$ disagree with a formal analytic continuation from $d \geq 2$. It, probably, is true also for the related interface and bulk critical exponents.

\section{References}

[1] N. M. Švrakic̀, V. Privman, D. B. Abraham, J. Stat. Phys. 53, 1041 (1988).

[2] L. J. Shaw, M. E. Fisher, Phys. Rev. A 39, 2189 (1989).

[3] M. P. Gelfand, M. E. Fisher, Physica A 166, 1 (1990).

[4] V. Privman, Phys. Rev. Lett. 61, 183 (1988).

[5] K. K. Mon, S. Wansleben, D. P. Landau, K. Binder, Phys. Rev. B 39, 7089 (1989).

[6] M. Hasenbusch, K. Pinn, Physica A 245, 366 (1997). 
[7] V. Privman, Int. J. Mod. Phys. C 3, 857 (1992).

[8] M. Hasenbusch, Int. J. Mod. Phys. C 12, 911 (2001).

[9] A. Bednorz, J. Phys. A 33, 5457 (2000).

[10] L. Landau, E. Lifshitz, Course of Theoretical Physics, Part 5: Statistical Physics, §141, Moscow, 1964.

[11] B. A. Berg, J. Stat. Phys. 82, 323 (1996).

[12] T. Koma, H. Tasaki, Phys. Rev. Lett. 74, 3916 (1995).

[13] Y. Gefen, B. B. Mandelbrot, A. Aharony, Phys. Rev. Lett. 45, 855 (1980).

[14] Rodney J. Baxter, Exactly Solved Models in Statistical Mechanics, Academic Press, London, 1989.

[15] J. A. Redinz, A. C. N. de Magelhaes, Phys. Rev. B 51, 2930 (1995). 